Article

\title{
Unification Theories: Means and Generalized Euler Formulas
}

\author{
Radu Iordanescu, Florin Felix Nichita *(D) and Ovidiu Pasarescu (D) \\ Simion Stoilow Institute of Mathematics of the Romanian Academy, 21 Calea Grivitei Street, \\ 010702 Bucharest, Romania; Radu.Iordanescu@imar.ro (R.I.); Ovidiu.Pasarescu@imar.ro (O.P.) \\ * Correspondence: florin.nichita@imar.ro; Tel.: +40-(0)-21-319-65-06; Fax: +40-(0)-21-319-65-05
}

Received: 16 November 2020; Accepted: 16 December 2020; Published: 20 December 2020

\begin{abstract}
The main concepts in this paper are the means and Euler type formulas; the generalized mean which incorporates the harmonic mean, the geometric mean, the arithmetic mean, and the quadratic mean can be further generalized. Results on the Euler's formula, the (modified) Yang-Baxter equation, coalgebra structures, and non-associative structures are also included in the current paper.
\end{abstract}

Keywords: means inequalities; Yang-Baxter equations; Euler's formula; coalgebra structures; non-associative algebras

MSC: 16T25; 17A01; 17B01; 17C50; 16T15; 26D07; 32A99; 00A35; 03B05; 06A05

\section{Introduction}

The current paper continues the study of unification theories, by examples and applications; according to [1], "it is essential, if mathematics is to stay as a whole and not become a pile of little bits of research, to provide a unification, which absorbs in some simple and general theories all the common substrata of the diverse branches (...). This is the good one can achieve with axiomatics".

The initial motivation of our study on means was a question about how to express the classical mean inequalities in a single formula. The generalized mean interpolates the harmonic mean, the geometric mean, the arithmetic mean, and the quadratic mean. It can be further generalized (using functions without critical points). Several new Euler type formulas were already proposed [2-6], and one could consider the common features of these formulas. Again a unification problem! Because some related representative coalgrebras were unified in previous papers, the answer to this question (without verbs, and formulated as an exclamation) was expected to be positive. Thus, we will unify the above versions of the Euler's formula in one theorem.

The current paper is organized as follows. After recalling the terminology on means, we present the functions $O, N$, and $M_{r}$ which interpolate the classical means. Their properties are not completely understood. Other basic inequalities are recommended (for undergraduate students). We think that behind these there might be deeper phenomena.

The next section refer to Euler's formula. There exist an Euler's formula for hyperbolic functions and an Euler's formula for dual numbers. All of these formulas are unified by a more general formula:

$$
e^{z J}=c(z)+J s(z)
$$

where $J$ is a matrix, and the functions $c$ and $s$ might not be cos and sin, but they behave like them, in the sense that they verify some trigonometric type identities. The colored Yang-Baxter equation is also related to this generalized Euler formula, and this fact is made more explicit in corollaries and a remark. 
Section 5 combines results on means inequalities, Euler's formula, non-associative algebras, and coalgebra structures. Classical means are extended for complex numbers. (At this point, the Euler's formula can be thought of as a mean.) We also discuss about a modified Yang-Baxter equation. The non-associative algebras results are related to a talk given at the 14th International Workshop on Differential Geometry and Its Applications, UPG Ploiesti, 2019. Remark 13 sustains the above statement on the unification of certain coalgebra structures.

A short section with commentaries will conclude this paper.

We will work over the fields $\mathbb{R}$ and $\mathbb{C}$. The tensor products will be defined over $\mathbb{C}$. As usual, we write $M_{n}(\mathbb{C})$ for the ring of all $n \times n$-matrices over the field $\mathbb{C}$. In particular, we write $I$ for the identity matrix in $M_{4}(\mathbb{C})$, respectively, $I^{\prime}$ for the identity matrix in $M_{2}(\mathbb{C})$.

\section{Classical Means-Terminology and Interpretations}

We first recall the definitions of some of the most used means from mathematics.

The harmonic mean: $H=H\left(a_{1}, \ldots, a_{n}\right)=\frac{n}{\frac{1}{a_{1}}+\ldots+\frac{1}{a_{n}}}, \quad a_{1}, \ldots, a_{n} \in \mathbb{R}^{*}$;

The geometric mean: $G=G\left(a_{1}, \ldots, a_{n}\right)=\sqrt[n]{a_{1} a_{2} \ldots a_{n}}, a_{1}, \ldots, a_{n} \in \mathbb{R}_{+}$;

The arithmetic mean: $A=A\left(a_{1}, \ldots, a_{n}\right)=\frac{a_{1}+\ldots+a_{n}}{n}, \quad a_{1}, \ldots, a_{n} \in \mathbb{R}$;

The quadratic mean: $Q=Q\left(a_{1}, \ldots, a_{n}\right)=\sqrt{\frac{a_{1}^{2}+\ldots+a_{n}^{2}}{n}}, \quad a_{1}, \ldots, a_{n} \in \mathbb{R}$;

The generalized mean: $m(\alpha)=m_{\alpha}\left(a_{1}, \ldots, a_{n}\right)=\sqrt[\alpha]{\frac{a_{1}^{\alpha}+\ldots+a_{n}^{\alpha}}{n}}, a_{1}, \ldots, a_{n} \in \mathbb{R}_{+}, \alpha \geq 1, \alpha \in \mathbb{N}$.

Remark 1. We note that $m(1)=A$ and $m(2)=Q$, for $a_{1}, \ldots, a_{n} \in \mathbb{R}_{+}$.

Remark 2. Let us consider the function (of $\alpha), m(\alpha)=\left(\frac{a_{1}^{\alpha}+\ldots+a_{n}^{\alpha}}{n}\right)^{\frac{1}{\alpha}}, \alpha \in \mathbb{R}^{*}$. One can extend the function $m(\alpha)$ to $\mathbb{R}$, by assigning $m(0)=m_{G}$. This is a natural extension because $\lim _{\alpha \rightarrow 0} m_{\alpha}\left(a_{1}, \ldots, a_{n}\right)=$ $m_{G}\left(a_{1}, \ldots, a_{n}\right)$.

Remark 3. It makes sense now to write: $m(0)=G$ and $m(-1)=H$. Thus, at this moment, we can say that the harmonic mean, the geometric mean, the arithmetic mean, and the quadratic mean are included in the generalized mean (for $a_{1}, \ldots, a_{n} \in \mathbb{R}_{+}$).

It is well-known that $m(\alpha)=\left(\frac{a_{1}^{\alpha}+\ldots+a_{n}^{\alpha}}{n}\right)^{\frac{1}{\alpha}}(m(0)=G)$ is an increasing function. In particular: $\cdots \leq m(-2) \leq m(-1)=m_{H} \leq m(0)=m_{G} \leq m(1)=m_{A} \leq m(2)=m_{Q} \leq m(3) \ldots$

Now, we consider the following function, which is a generalization of the above function $m(\alpha)$.

Theorem 1. Let $O: \mathbb{R} \times \mathbb{R} \rightarrow \mathbb{R}$, be defined by the folowing rule:

$$
O(x, y)=\left(\frac{a_{1}^{x}+\ldots+a_{n}^{x}}{a_{1}^{y}+\ldots+a_{n}^{y}}\right)^{\frac{1}{x-y}} \text { for } x \neq y, \quad O(x, x)=e^{\frac{a_{1}^{x} \ln a_{1}+\ldots+a_{n}^{x} \ln a_{n}}{a_{1}^{x}+\ldots+a_{n}^{x}}} \text {. }
$$

Then, the function $O$ has the following properties:

1. It is a continuous function.

2. $O(x, y)=O(y, x) \quad \forall x, y \in \mathbb{R}$

3. For any fixed $x_{0} \in \mathbb{R}$, the function $f(y)=O\left(x_{0}, y\right)$ is a strongly increasing function.

4. For any fixed $y_{0} \in \mathbb{R}$, the function $g(x)=O\left(x, y_{0}\right)$ is a strongly increasing function.

Remark 4. We can easily see that: $O(-1,0)=H, O(0,0)=G, O(1,0)=A, O(2,0)=Q$, and, in general, $O(\alpha, 0)=m(\alpha)$.

In addition, one can show that: $O(x, x)=e^{\frac{a_{1}^{x} \ln a_{1}+\ldots+a_{n}^{x} \ln a_{n}}{a_{1}^{x}+\ldots+a_{n}^{x}}}=a_{1}^{\frac{a_{1}^{x}}{a_{1}^{x}+\ldots+a_{n}^{x}}} \ldots a_{n}^{\frac{a_{1}^{x}+\ldots+a_{n}^{x}}{a^{x}}}$ (it follows more clearly now that $O(0,0)=G)$.

Proof of Theorem 1. We now prove the first claim. We denote $r:=x-y$. 


$$
\begin{aligned}
& \lim _{x \rightarrow y}\left(\frac{a_{1}^{x}+\ldots+a_{n}^{x}}{a_{1}^{y}+\ldots+a_{n}^{y}}\right)^{\frac{1}{x-y}}= \\
& \lim _{r \rightarrow 0}\left[\left(1+\frac{a_{1}^{y}\left(a_{1}^{r}-1\right)+\ldots+a_{n}^{y}\left(a_{n}^{r}-1\right)}{a_{1}^{y}+\ldots+a_{n}^{y}}\right) \frac{a_{1}^{y}+\ldots+a_{n}^{y}}{a_{1}^{y}\left(a_{1}^{r}-1\right)+\ldots+a_{n}^{y}\left(a_{n}^{r}-1\right)}\right] \\
& \frac{a_{1}^{y} \frac{a_{1}^{r}-1}{r}+\ldots+a_{n}^{y} \frac{a_{n}^{r}-1}{r}}{a_{1}^{y}+\ldots+a_{n}^{y}}
\end{aligned}
$$

This proves the continuity.

We will jump now to the third claim. (The proofs of 2 and 4 will then be immediate).

For any fixed $x_{0} \in \mathbb{R}$, we will prove that $f^{\prime}(y)=\frac{\partial}{\partial y} O\left(x_{0}, y\right)>0, \forall y \in \mathbb{R}-\left\{x_{0}\right\}$. This implies that $f(y)$ is increasing on $\left(-\infty, x_{0}\right)$ and $\left(x_{0}, \infty\right)$ and, being continuous in $\left(x_{0}, x_{0}\right)$, it follows that it is an increasing function on $\mathbb{R}$.

We will differentiate $\ln f(y)=\frac{\ln \left(a_{1}^{y}+\ldots+a_{n}^{y}\right)-\ln \left(a_{1}^{x}+\ldots+a_{n}^{x}\right)}{y-x}$; thus, we get

$$
\frac{f^{\prime}(y)}{f(y)}=\frac{\frac{\left(a_{1}^{y} \ln a_{1}+\ldots+a_{n}^{y} \ln a_{n}\right.}{\left(a_{1}^{y}+\ldots+a_{n}^{y}\right.}(y-x)-\left[\ln \left(a_{1}^{y}+\ldots+a_{n}^{y}\right)-\ln \left(a_{1}^{x}+\ldots+a_{n}^{x}\right)\right]}{(y-x)^{2}} \text {. }
$$

Let us denote $\alpha(t)=\ln \left(a_{1}^{t}+\ldots+a_{n}^{t}\right)$.

Then, by the Lagrange Theorem, $\alpha(y)-\alpha(x)=(y-x) \alpha^{\prime}(\zeta), \zeta \in(x, y)$; thus,

$$
f^{\prime}(y)=f(y) \frac{\frac{a_{1}^{y} \ln a_{1}+\ldots+a_{n}^{y} \ln a_{n}}{a_{1}^{y}+\ldots+a_{n}^{y}}(y-x)-\frac{a_{1}^{\zeta} \ln a_{1}+\ldots+a_{n}^{\zeta} \ln a_{n}}{a_{1}^{y}+\ldots+a_{n}^{y}}(y-x)}{(y-x)^{2}}=f(y) \frac{\frac{a_{1}^{y} \ln a_{1}+\ldots+a_{n}^{y} \ln a_{n}}{a_{1}^{y}+\ldots+a_{n}^{y}}-\frac{a_{1}^{\zeta} \ln a_{1}+\ldots+a_{n}^{\zeta} \ln a_{n}}{a_{1}^{y}+\ldots+a_{n}^{y}}}{(y-x)}
$$

Let $u(t)=\frac{a_{1}^{t} \ln a_{1}+\ldots+a_{n}^{t} \ln a_{n}}{a_{1}^{t}+\ldots+a_{n}^{t}}$; so, $f^{\prime}(y)=f(y) \frac{u(y)-u(\zeta)}{y-x}$, with $\zeta \in(x, y)$.

It is enough to prove that $\mathrm{u}(\mathrm{t})$ is an increasing function.

However, $u^{\prime}(t)=\frac{\left(a_{1}^{t} \ln ^{2} a_{1}+\ldots+a_{n}^{t} \ln ^{2} a_{n}\right)\left(a_{1}^{t}+\ldots+a_{n}^{t}\right)-\left(a_{1}^{t} \ln a_{1}+\ldots+a_{n}^{t} \ln a_{n}\right)^{2}}{\left(a_{1}^{t}+\ldots+a_{n}^{t}\right)^{2}}$, and it is greater than or equal to zero by the Cauchy-Schwarz inequality.

\section{Means Unification and New Inequalities}

Definition 1. For strictly positive real numbers $a_{j}, 1 \leq j \leq n+1$, we consider the real function

$$
N: \mathbb{R}^{n} \rightarrow \mathbb{R}, \quad N\left(x_{1}, \ldots, x_{n}\right)=\frac{a_{1}^{x+1}+\cdots+a_{n+1}^{x+1}}{a_{2}^{x_{1}} \ldots a_{n+1}^{x_{n}}+a_{3}^{x_{1}} \ldots a_{n+1}^{x_{n-1}} a_{1}^{x_{n}}+\cdots+a_{1}^{x_{1}} a_{2}^{x_{2}} \ldots a_{n}^{x_{n}}}
$$

where we have used the notation $x:=x_{1}+\cdots+x_{n}$.

Remark 5. We can see that:

$$
N(0, \ldots, 0)=A, N\left(\frac{-1}{n+1}, \ldots, \frac{-1}{n+1}\right)=G, N(-1,0, \ldots, 0)=H .
$$

The properties of the fuction $N\left(x_{1}, \ldots, x_{n}\right)$, for the case $n=2$, were presented in [4].

We will restict our study to the case of means of two numbers in the remaining of this section. We will introduce additional notation below.

Remark 6. For three real numbers $a>0, b>0$, and $r>0$, the real function $M_{r}: \mathbb{R} \rightarrow \mathbb{R}, \quad M_{r}(x)=\left(\frac{a^{x}+b^{x}}{a^{x-r}+b^{x-r}}\right)^{\frac{1}{r}}$ is an increasing function.

One way to prove this fact is by direct computations. Alternatively, the proof follows as a particular case of Theorem 1. The function $M_{r}$ is also related to the function $N\left(x_{1}, \ldots, x_{n}\right)$ from the Definition 1.

What is the relationship between $M_{r}(x)$ and $M_{p}(y)$ ? A tentative answer is given below. 
Theorem 2. The following inequality for real numbers, where $a>0, b>0$, and $x \leq y$,

$$
M_{r}(x)=\left(\frac{a^{x}+b^{x}}{a^{x-r}+b^{x-r}}\right)^{\frac{1}{r}} \leq\left(\frac{a^{y}+b^{y}}{a^{y-p}+b^{y-p}}\right)^{\frac{1}{p}}=M_{p}(y)
$$

holds for the following additional conditions:

(i) $p=r_{1}$

(ii) $p=\frac{1}{2}, r=1, x=y$;

(iii) $p=\overline{1}^{\prime}, r=\frac{1}{2}$ and $x+\frac{1}{2}=y$.

Proof. A direct proof is suggested.

We now present inequalities which follow directly from the above Theorem 2 ( for $a>0, b>0$ ):

$$
\frac{2}{\frac{1}{a}+\frac{1}{b}} \leq\left(\frac{2}{\frac{1}{\sqrt{a}}+\frac{1}{\sqrt{b}}}\right)^{2} \leq \sqrt{a b} \leq\left(\frac{\sqrt{a}+\sqrt{b}}{2}\right)^{2} \leq \frac{a+b}{2} \leq a+b-\sqrt{a b} \leq \frac{a^{2}+b^{2}}{a+b}
$$

More demanding are the inequalities given below $(a>0, b>0)$ :

$$
\frac{a+b}{2} \leq\left(\frac{a^{\frac{3}{2}}+b^{\frac{3}{2}}}{2}\right)^{\frac{2}{3}} \leq \sqrt{\frac{a^{2}+b^{2}}{2}} \leq a+b-\sqrt{a b}
$$

It could be an interesting exercise for undergraduate students to prove the above inequalities using only basic algebra.

Remark 7. Note also the following types of inequalities for means $(a>0, b>0)$ :

$$
\sqrt{a b} \leq \frac{\frac{a+b}{2}+\sqrt{a b}+\frac{2}{\frac{1}{a}+\frac{1}{b}}}{3} \leq \frac{\frac{a+b}{2}+\frac{2}{\frac{1}{a}+\frac{1}{b}}}{2}
$$

Again, it is surprising that the above inequalities can be proved by hand. They could be part of a Calculus homework.

Remark 8. It is an open problem to prove that, for $p \geq r, M_{r}(x) \leq M_{p}(x) \forall x \in \mathbb{R}$.

In addition, we conjecture that $M_{r}(x) \leq M_{p}(y) \forall x-r \leq y-p$.

Future investigations will try to extend the conditions $(i-i i i)$ in the above Theorem 2 , and to prove versions of the above inequalities with more variables.

\section{Generalized Euler Formula}

In this section, we will identify linear applications with matrices, without explicitly mentioning this fact. The exponential function of a matrix is thought as the Taylor expansion of the real exponential function evaluated at the given matrix.

Theorem 3. Let $J \in M_{n}(\mathbb{C})$ be such that

$$
\left(J \otimes I^{\prime}\right) \circ\left(I^{\prime} \otimes J\right)=\left(I^{\prime} \otimes J\right) \circ\left(J \otimes I^{\prime}\right)
$$

If $e^{z J}=R(z), z \in \mathbb{C}$, then the colored Yang-Baxter equation is satisfied:

$$
\left(R \otimes I^{\prime}\right)(z) \circ\left(I^{\prime} \otimes R\right)(z+w) \circ\left(R \otimes I^{\prime}\right)(w)=\left(I^{\prime} \otimes R\right)(w) \circ\left(R \otimes I^{\prime}\right)(z+w) \circ\left(I^{\prime} \otimes R\right)(z) .
$$


Proof. The proof will use the property (5) and the formula $R(z)=e^{z J}=\sum_{0}^{\infty} \frac{z^{n}}{n !} J^{n}$. One can now reduce the condition (6) to a simpler relation, $z\left(J \otimes I^{\prime}\right)+(z+w)\left(I^{\prime} \otimes J\right)+w\left(J \otimes I^{\prime}\right)=$ $w\left(I^{\prime} \otimes J\right)+(z+w)\left(J \otimes I^{\prime}\right)+z\left(I^{\prime} \otimes J\right)$, which is easily verified.

Theorem 4. For $J \in M_{n}(\mathbb{C}), J^{2}=\alpha I(\alpha \in \mathbb{C})$, there exist two functions $c, s: \mathbb{C} \rightarrow \mathbb{C}$, $c(z+w)=c(z) c(w)+\alpha s(z) s(w), \quad s(z+w)=s(z) c(w)+c(z) s(w)$, such that:

$$
e^{z J}=c(z) I+s(z) J .
$$

The functions $c$ and s could have the following forms:

(i) if $\alpha=0$ in the above case, then $c(z)=1$ and $s(z)=z$ ín or $\quad c(z)=0$ and $s(z)=0$;

(ii) if $\alpha=\beta^{2} \neq 0$, then $c(z)=\cosh (\beta z)$ and $s(z)=\frac{\sinh (\beta z)}{\beta}$.

Proof. We will now prove the general statement. Because $e^{z J}=\sum_{0}^{\infty} \frac{z^{n}}{n !} J^{n}$ and $J^{2}=\alpha I$ (with $\alpha \in \mathbb{C}$ ), it follows that $e^{z J}=c(z) I+s(z) J$ for some complex functions $c, s: \mathbb{C} \rightarrow \mathbb{C}$.

We want to show that the functions $c$ and $s$ have the above properties. However, $e^{z J} e^{w J}=e^{(z+w) J}$ implies that $(c(z) I+s(z) J)(c(w) I+s(w) J)=c(z+w) I+s(z+w) J$.

The condition $(c(z) c(w)+\alpha s(z) s(w)) I+(s(z) c(w)+c(z) s(w)) J=c(z+w) I+s(z+w) J$ implies the properties of $c$ and $s$.

The last part of the proof is based on the analysis of the terms of the series $e^{z J}=\sum_{0}^{\infty} \frac{z^{n}}{n !} J^{n}$. The case $\beta=0$ is included in (i). It is easier now to check directly that the sum of the functions given in (ii) is equal to $e^{z J}$.

Remark 9. If $J=i$ I, formula (7) is equivalent with $e^{i x}=\cos x+i \sin x$.

Corollary 1. If we consider the complex valuated matrix $J=\left(\begin{array}{ll}0 & V \\ 0 & 0\end{array}\right)$, where $V=\left(\begin{array}{ll}c & d \\ 0 & c\end{array}\right)(c, d \in \mathbb{C})$, then $J^{2}=0_{4}, \quad\left(J \otimes I^{\prime}\right) \circ\left(I^{\prime} \otimes J\right)=\left(I^{\prime} \otimes J\right) \circ\left(J \otimes I^{\prime}\right), I+J x=e^{x J}=R(x)$ and

$$
\left(R \otimes I^{\prime}\right)(x) \circ\left(I^{\prime} \otimes R\right)(x+y) \circ\left(R \otimes I^{\prime}\right)(y)=\left(I^{\prime} \otimes R\right)(y) \circ\left(R \otimes I^{\prime}\right)(x+y) \circ\left(I^{\prime} \otimes R\right)(x) .
$$

Corollary 2. If $J=\left(\begin{array}{cc}0 & W \\ X & 0\end{array}\right)$, where $W=\left(\begin{array}{ll}0 & 1 \\ i & 0\end{array}\right)$ and $X=\left(\begin{array}{cc}0 & i \\ -1 & 0\end{array}\right)$, then $e^{z J}=\cos (z) I+\sin (z) J$, which is a solution for (6).

Corollary 3. If $J=\left(\begin{array}{cc}0 & U \\ U & 0\end{array}\right)$, where $U=\left(\begin{array}{ll}0 & 1 \\ 1 & 0\end{array}\right)$, then $e^{z J}=\cosh (z) I+\sinh (z) J$; this is also a solution for (6).

\section{Means, Euler Formulas, Non-Associative Algebras, and Coalgebra Structures}

After a shor digression on the Euler's formula and the Yang-Baxter equation, we are back to means, in an attempt to extend their domain to the complex numbers (using Euler's formula).

Theorem 5. For $z, w \in \mathbb{C}$, such that $z=\rho e^{i \alpha}, w=\rho^{\prime} e^{i \beta}$, with $\alpha, \beta \in[0,2 \pi)$, we define:

$$
A(z, w)=\frac{z+w}{2}, \quad G(z, w)=\sqrt{\rho \rho^{\prime}} e^{i \frac{\alpha+\beta}{2}}, \quad H(z, w)= \begin{cases}\frac{2 z w}{z+w}, & \text { if } z \neq-w \\ 0, & \text { otherwise }\end{cases}
$$

If $\alpha=\beta$ or $\rho_{\text {Max }} \geq 7 \rho_{\min }$, then:

$$
\|H\| \leq\|G\| \leq\|A\|
$$


Proof. For $\alpha=\beta$, the inequalities $\|H\| \leq\|G\| \leq\|A\|$ are equivalent to the classical means inequalities.

The inequality $\|G\| \leq\|A\|$ is equivalent to finding $x \in \mathbb{R}$ such that $x^{2}+2[\cos (\alpha-\beta)-2] x+1 \geq 0$.

The last part of the theorem is left for the interested reader. It also will be treated in detail in the nearest future.

Remark 10. For $z, w \in \mathbb{C}$, we define:

$$
Q(z, w)=\frac{z+i w}{\sqrt{2}}
$$

For $\alpha=\beta$, the inequalities $\|H\| \leq\|G\| \leq\|A\| \leq\|Q\|$ are equivalent to the usual inequalities for means.

For $A \in M_{n}(\mathbb{C})$ and $D \in M_{n}(\mathbb{C})$ being a diagonal matrix, we proposed (see [7]) the problem of finding $X \in M_{n}(\mathbb{C})$ such that

$$
A X A+X A X=D
$$

Equation (11) is a type of Yang-Baxter matrix equation if $D=O_{n}$ and $X=-Y$. It is related to the three matrix problem, and it can be interpretated as "a generalized eigenvalue problem".

For $A \in M_{2}(\mathbb{C})$, there is a matrix with trace -1 , and

$$
D=-\left(\begin{array}{cc}
\operatorname{det}(A) & 0 \\
0 & \operatorname{det}(A)
\end{array}\right)
$$

Equation (11) has a solution $\mathrm{X}=\mathrm{I}^{\prime}$.

Remark 11. If $A=\left(J \otimes I^{\prime}\right)$ and $X=\left(I^{\prime} \otimes J\right)$ as in formula (5), then (11) reduces to $\left(J^{2} \otimes I^{\prime}\right)\left(I^{\prime} \otimes J\right)+\left(J \otimes I^{\prime}\right)\left(I^{\prime} \otimes J^{2}\right)=D$.

If $J^{2}=0$, we obtain a solution; in particular, for $J=\left(\begin{array}{ll}0 & V \\ 0 & 0\end{array}\right)$, where $V=\left(\begin{array}{ll}c & d \\ 0 & c\end{array}\right), c, d \in \mathbb{C}$, the pair $A=\left(J \otimes I^{\prime}\right)$ and $X=\left(I^{\prime} \otimes J\right)$ is a solution for (11).

Definition 2. For $a \mathbb{C}$-space $V$, let $\eta: V \otimes V \rightarrow V, a \otimes b \mapsto a b$, be a linear map such that:

$$
\begin{gathered}
(a b) c+(b c) a+(c a) b=a(b c)+b(c a)+c(a b) \\
\left(a^{2} b\right) a=a^{2}(b a), \quad(a b) a^{2}=a\left(b a^{2}\right), \quad\left(b a^{2}\right) a=(b a) a^{2}, \quad a^{2}(a b)=a\left(a^{2} b\right),
\end{gathered}
$$

$\forall a, b, c \in V$. Then, $(V, \eta)$ is called a UJLA structure.

Remark 12. If $(A, \theta)$, where $\theta: A \otimes A \rightarrow A, \theta(a \otimes b)=a b$, is a (non-unital) associative algebra over $\mathbb{C}$, then we define a UJLA structure $\left(A, \theta^{\prime}\right)$, where $\theta^{\prime}(a \otimes b)=a b \cos \alpha+b a \sin \alpha$, for $\alpha \in \mathbb{R}$. For $\alpha=\frac{\pi}{4}$, $\left(A, \theta^{\prime}\right)$ is a Jordan algebra; for $\alpha=\frac{3 \pi}{4},\left(A, \theta^{\prime}\right)$ is a Lie algebra.

Theorem 6. If $(A, \theta)$, where $\theta: A \otimes A \rightarrow A, \theta(a \otimes b)=a b$, is a (non-unital) associative algebra over $\mathbb{C}$, then we define a UJLA structure $\left(A, \theta^{\prime}\right)$, where $\theta^{\prime}(a \otimes b)=\theta_{\alpha}^{\prime}(a \otimes b)=a b \cos \alpha+i b a \sin \alpha$, for $\alpha \in \mathbb{C}$. The following properties happen:

(i) If $\alpha=\frac{\pi}{2}$, then $\left(A_{,}(-i) \theta^{\prime}\right)$ is just the opposite algebra (and if $\alpha=0$, then $\theta^{\prime}(a \otimes b)=\theta(a \otimes b)$ ).

(ii) For the product $\theta_{\alpha}^{\prime}$ and $\beta \in \mathbb{C}$, we associate another product $\theta_{\beta}^{\prime \prime}(a \otimes b)=\theta_{\alpha}^{\prime}(a \otimes b) \cos \beta+\theta_{\alpha}^{\prime}(b \otimes$ a) $\sin \beta$. Then, $\theta_{\beta}^{\prime \prime}(a \otimes b)=\theta_{\alpha+\beta}^{\prime}(a \otimes b)=a b \cos (\alpha+\beta)+i b a \sin (\alpha+\beta)$.

(iii) If the initial algebra is commutative, then $\theta^{\prime}(a \otimes b)=\theta_{\alpha}^{\prime}(a \otimes b)=e^{i \alpha} a b$.

Proof. The statements (i) and (iii) can be proved directly. For (ii), we observe that $\theta_{\alpha}^{\prime}(a \otimes b) \cos \beta+$ $\theta_{\alpha}^{\prime}(b \otimes a) \sin \beta=(a b \cos \alpha+i b a \sin \alpha) \cos \beta+i(b a \cos \alpha+i a b \sin \alpha) \sin \beta=a b \cos \alpha \cos \beta-$ 
$a b \sin \alpha \sin \beta+i b a \sin \alpha \cos \beta+i b a \cos \alpha \sin \beta=a b(\cos \alpha \cos \beta-\sin \alpha \sin \beta)+i b a(\sin \alpha \cos \beta+$ $\cos \alpha \sin \beta)=a b \cos (\alpha+\beta)+i b a \sin (\alpha+\beta)$.

Theorem 7. For $(V, \eta)$ a UJLA structure, $D(x)=D_{b}(x)=b x-x b$ is a UJLA-derivation (i.e., $D\left(a^{2} a\right)=$ $\left.D\left(a^{2}\right) a+a^{2} D(a) \forall a \in V\right)$.

Proof. In Formula (13), we take $c=a^{2}:(a b) a^{2}+\left(b a^{2}\right) a+\left(a^{2} a\right) b=a\left(b a^{2}\right)+b\left(a^{2} a\right)+a^{2}(a b)$. It follows from (14) that $\left(b a^{2}\right) a+\left(a^{2} a\right) b=b\left(a^{2} a\right)+a^{2}(a b)$.

Thus, $\left(b a^{2}\right) a-a^{2}(a b)=b\left(a^{2} a\right)-\left(a^{2} a\right) b$; so, $b\left(a^{2} a\right)-\left(a^{2} a\right) b=\left(b a^{2}-a^{2} b\right) a+a^{2}(b a-a b)$. Thus, $D\left(a^{2} a\right)=D\left(a^{2}\right) a+a^{2} D(a)$.

Remark 13. Formula (7) can be interpretated in terms of coalgebras (see $[7,8])$. There exists a coalgebra $\frac{\mathbb{C}[X]^{2}}{X}=\mathbb{C}[a]$, where $a^{2}=\alpha=\beta^{2} \in \mathbb{C}$, generated by two generators $c$ and $s$, such that:

$$
\Delta(c)=u \otimes u+\alpha s \otimes s, \Delta(s)=c \otimes s+s \otimes c, \varepsilon(c)=1, \varepsilon(s)=0 .
$$

Now, formula (7) leads to the subcoalgebra generated by $u+\beta s$.

For $\beta=i$, we obtain the trigonometric coalgebra. (It was shown by S. Catoiu that it can be extended to a bialgebra.) The subcoalgebra generated by $c+$ is related to the Euler's formula.

For $\beta=1$, we obtain a coalgebra generated by $c$ and $s$, such that $\Delta(c)=u \otimes u+s \otimes s, \Delta(s)=$ $c \otimes s+s \otimes c, \varepsilon(c)=1$ and $\varepsilon(s)=0$. There exists a subcoalgebra generated by $c+s$, related to the Euler formula for hyperbolic functions.

\section{Final Comments and Implications}

At the end of this trip through several branches of mathematics, we will add a few words. As we continued our work on unification theories begun in previous papers (see, for example, $[2,4,7,9,10]$ ), the unification approaches led us to new results. The Yang-Baxter equation (see [11-14]) can be interpreted in terms of logical circuits and, in logic, it represents a kind of compatibility condition when working with many logical sentences at the same time. In this type of logic, we work at the same time with sentences that contradict each other. This discussion will be continued later, and will be also related to literature.

Author Contributions: The authors met at workshops, had private meetings, and published related papers; supervision, R.I.; Section 2 is entirely due to O.P.; validation, R.I., O.P. and F.F.N. All authors have read and agreed to the published version of the manuscript.

Funding: This research received no external funding.

Acknowledgments: The authors are grateful for the support received from the Referees, the Editors and the Editorial Staff.

Conflicts of Interest: The authors declare no conflict of interest.

\section{References}

1. Wikiquote-Andre Weil. Available online: https://en.wikiquote.org/wiki/Andre_Weil (accessed on 18 December 2020).

2. Marcus, S.; Nichita, F.F. On Transcendental Numbers: New Results and a Little History. Axioms 2018, 7, 15. [CrossRef]

3. Ludkowski, S.V. Octonion Measures for Solutions of PDEs. Adv. Appl. Clifford Algebr. 2020, 30, 39. [CrossRef]

4. Nichita, F.F. Mathematics and Poetry · Unification, Unity, Union. Science 2020, 2, 72. [CrossRef]

5. Ludkovsky, S.V. Differentiable functions of Cayley-Dickson numbers and line integration. J. Math. Sci. 2007, 141, 1231-1298. [CrossRef]

6. Ludkovsky, S.V.; van Oystaeyen, F. Differentiable functions of quaternion variables. Bull. Sci. Math. 2003, 127, 755-796. [CrossRef]

7. Nichita, F.F. Unification Theories: Examples and Applications. Axioms 2018, 7, 85. [CrossRef]

8. Majid, S. A Quantum Groups Primer; Cambridge University Press: Cambridge, UK, 2002. 
9. Nichita, F.F. Unification Theories: New Results and Examples. Axioms 2019, 8, 60. [CrossRef]

10. Iordanescu, R.; Nichita, F.F.; Nichita, I.M. The Yang-Baxter Equation, (Quantum) Computers and Unifying Theories. Axioms 2014, 3, 360-368. [CrossRef]

11. Oner, T.; Senturk, I.; Oner, G. An Independent Set of Axioms of MV-Algebras and Solutions of the SetTheoretical Yang-Baxter Equation. Axioms 2017, 6, 17. [CrossRef]

12. Oner, T.; Katican, T. On Solutions to the Set-Theoretical Yang-Baxter Equation in Wajsberg-Algebras. Axioms 2018, 7, 6. [CrossRef]

13. Nichita, F.F. (Ed.) Hopf Algebras, Quantum Groups and Yang-Baxter Equations; MDPI: Basel, Switzerland, 2019. ISBN1 978-3-03897-324-9 (Pbk), ISBN2 978-3-03897-325-6 (PDF).

14. Oner, T.; Katican, T. Interval Sheffer Stroke Basic Algebras and Yang-Baxter Equation, preprint, February 2020.

Publisher's Note: MDPI stays neutral with regard to jurisdictional claims in published maps and institutional affiliations.

(C) 2020 by the authors. Licensee MDPI, Basel, Switzerland. This article is an open access article distributed under the terms and conditions of the Creative Commons Attribution (CC BY) license (http:/ / creativecommons.org/licenses/by/4.0/). 